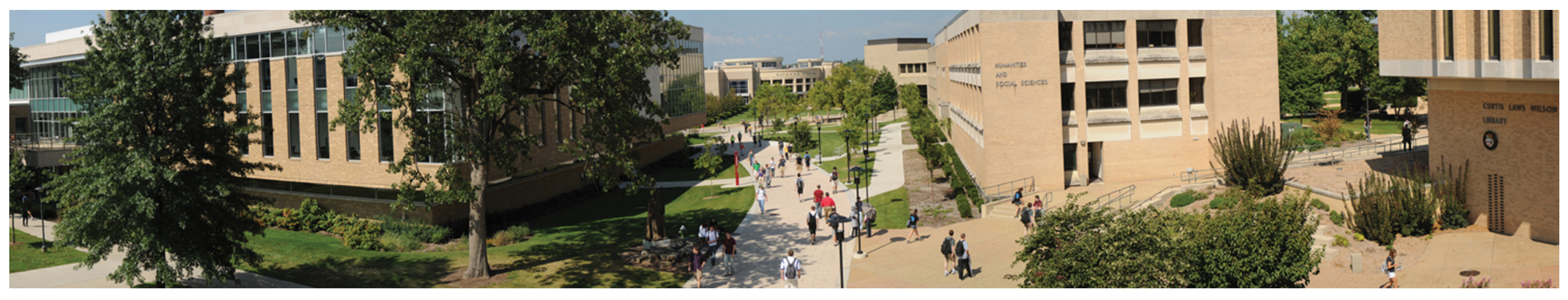

\title{
Acoustic Radiation from a Mach 14 Turbulent Boundary Layer
}

\section{Chao Zhang and Lian Duan}

Missouri University of Science and Technology

Rolla, MO 65409

Meelan Choudhari

NASA Langley Research Center

Hampton, VA 23693

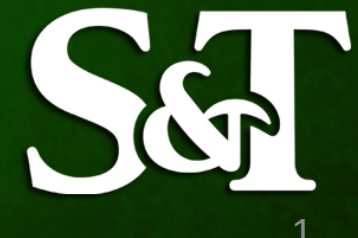


- Transition testing in hypersonic ground facilities

- an important avenue to understanding the laminar-turbulent transition behavior of hypersonic vehicles

- Most hypersonic wind tunnels have elevated freestream disturbances

- Tunnel Disturbances have a large impact on Transition at $M>1$
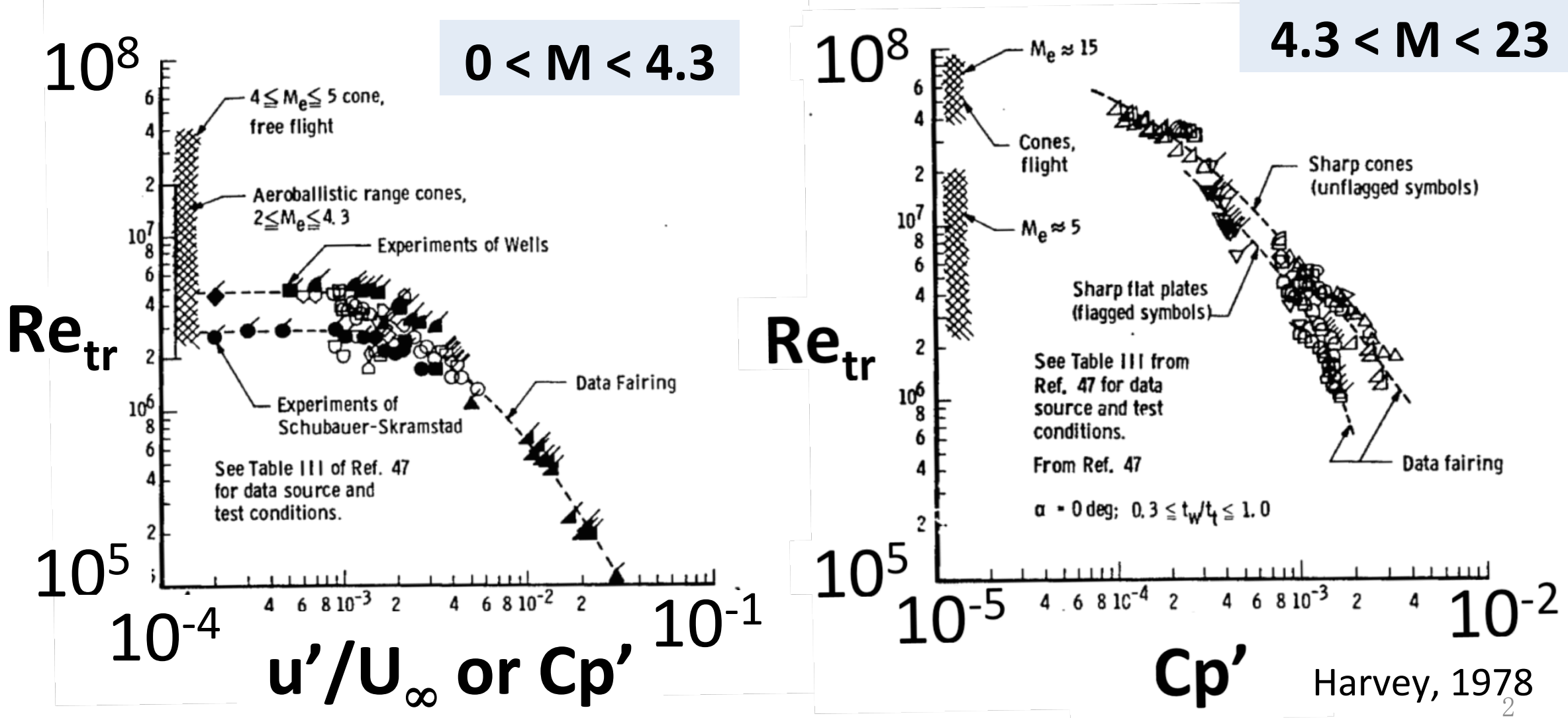


\section{Background}

Missouri University of Science and Technology

Disturbance Environment for Wind-Tunnel Facilities

(Blanchard et al. 1997)

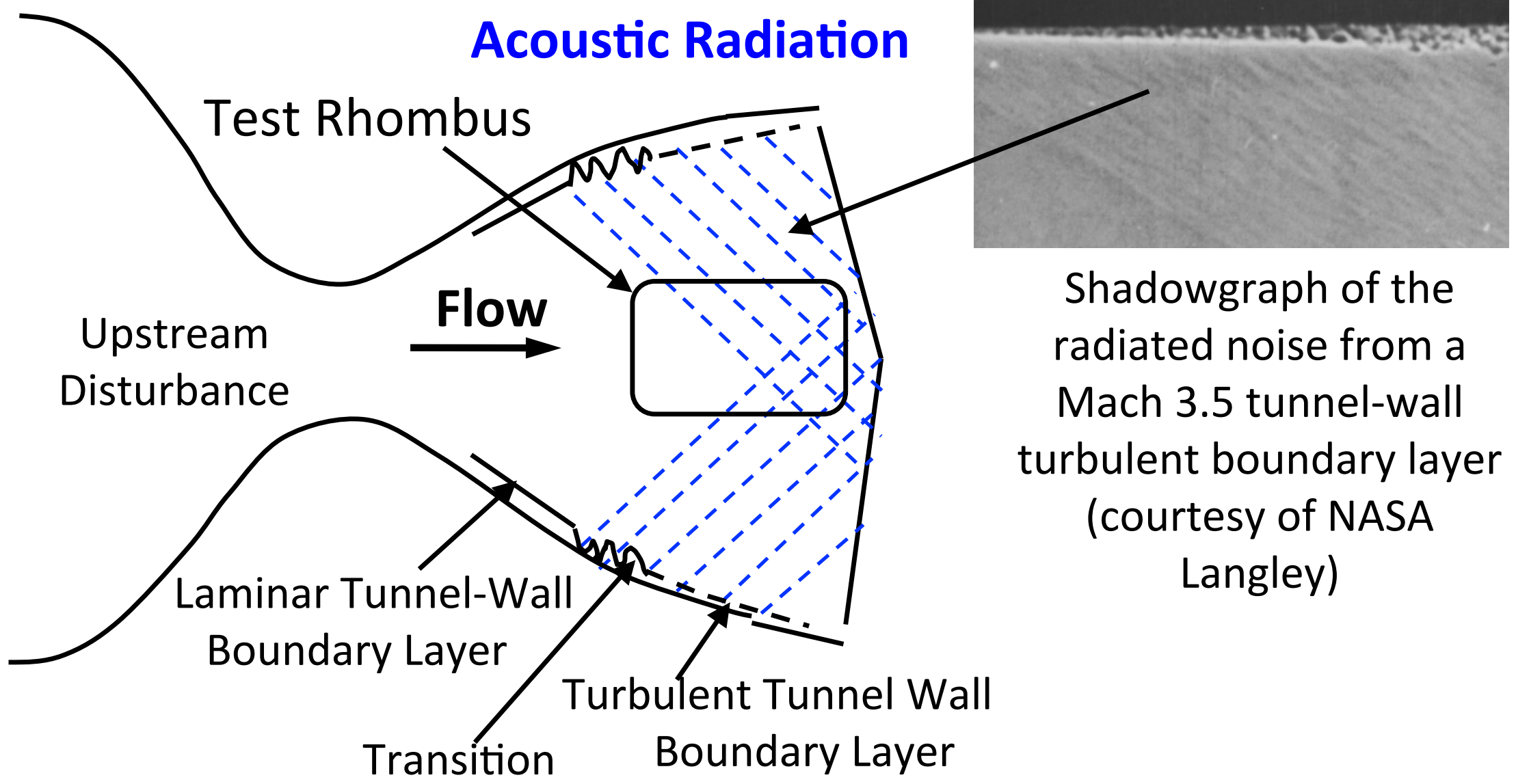

In a conventional ("noisy") tunnel, tunnel disturbances dominated by acoustic radiation from tunnel wall turbulent boundary layers for $M>\mathbf{2 . 5}$ (Laufer, 1964) 


\section{Methodology}

\section{Approach}

High-fidelity simulation of acoustic radiation from tunnel-wall turbulent boundary layers

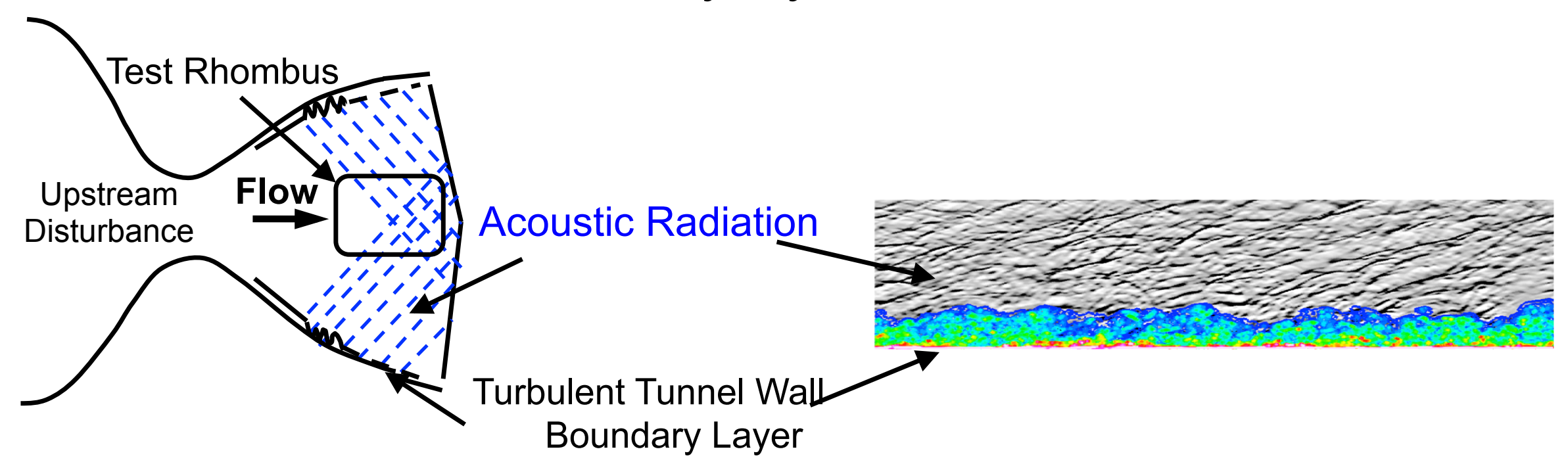

Impact: Understanding the acoustic fluctuations in wind tunnels and their influence on boundary layer transition would enable

- Better use of transition data

- Meaningful application of receptivity theory (Fedorov and Khokhlov, 1991)

- Potential reconciliation of differences in transition onset across multiple facilities 


\section{Acoustic Radiation from High-Speed Turbulent BLs}

\section{Theory}

- Eddy Mach wave convecting supersonically with respect to free stream (Phillips, 1960; Ffowcs-Williams \& Maidanik 1963)

- Restricted to prediction of intensity of the freestream fluctuation Experiments

- Laufer (1961, 1964); Kendall (1970); Rufer (2000); Bounitch et al. (2011); Masutti et al. (2013); Radespiel et al. (2013)

- Mostly limited to amplitude and spectra with limited bandwidth; no multi-point statistics 


\section{Acoustic Radiation from High-Speed Turbulent BLS}

Direct Numerical Simulations (Duan et al., AIAA 2012-3070, AIAA 2013-0532, AIAA 2014-2912, JFM vol. 746, pp 165-192, 2014 )

- include both the flow field and near-acoustic field

- isolate a purely acoustic freestream disturbance field above a single tunnel wall

- Identify generic statistical and spectral features of freestream disturbances

- Open doors to further simulations of receptivity in a tunnel-like environment DNS datasets:

$-\mathrm{M}_{\infty}=2.5, \mathrm{~T}_{\mathrm{w}} / \mathrm{T}_{\mathrm{r}}=1.0$, Flat Plate

$-M_{\infty}=5.86, T_{w} / T_{r}=0.76$, Flat Plate $(M 6 T w 076) \& T_{w} / T_{r}=0.25$, Flat Plate (M6Tw025)

- Freestream condition representative of Purdue Quiet Tunnel under noisy condition with $\mathrm{p}_{0}=132 \mathrm{psi}, \mathrm{T}_{0}=432 \mathrm{~K}$

$-\mathbf{M}_{\infty}=14, \mathrm{~T}_{\mathrm{w}} / \mathrm{T}_{\mathrm{r}}=\mathbf{0 . 1 8}(\mathrm{M} 14 \mathrm{Tw} 018)$ Flat Plate

- Freestream condition representative of AEDC Tunnel 9 at $p_{0}=1,023$ psi

- Comparison with Boundary-layer measurements at AEDC Tunnel 9 (Expected) 


\section{Comparison with Experiment (M6Tw076)}
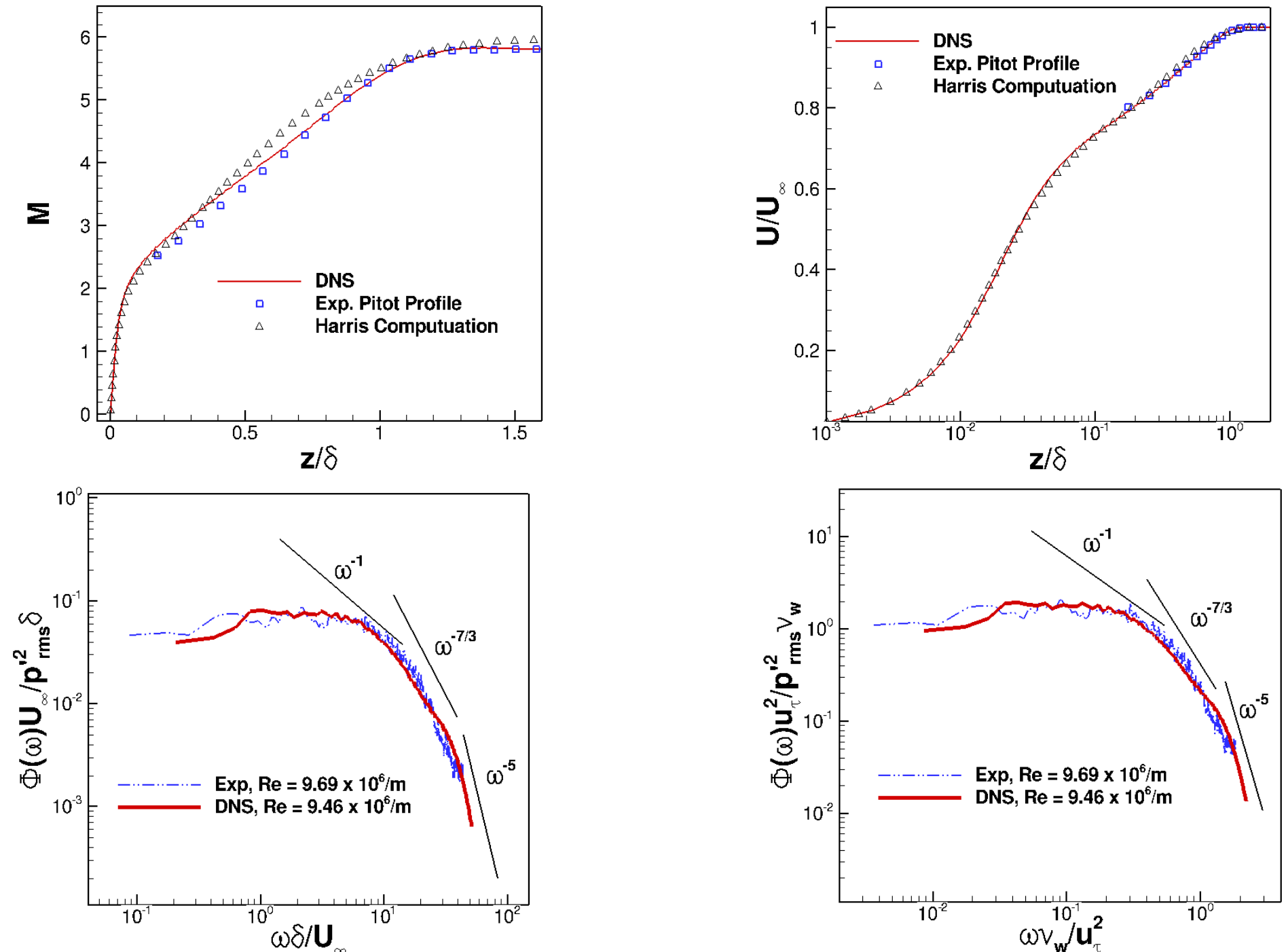

Mean flow predictions and wall-p' frequency spectrum are in good agreement with the measurements in the Boeing/AFOSR Mach 6 Quiet Tunnel under noisy condition 


\section{Normalized Frequency Spectra}
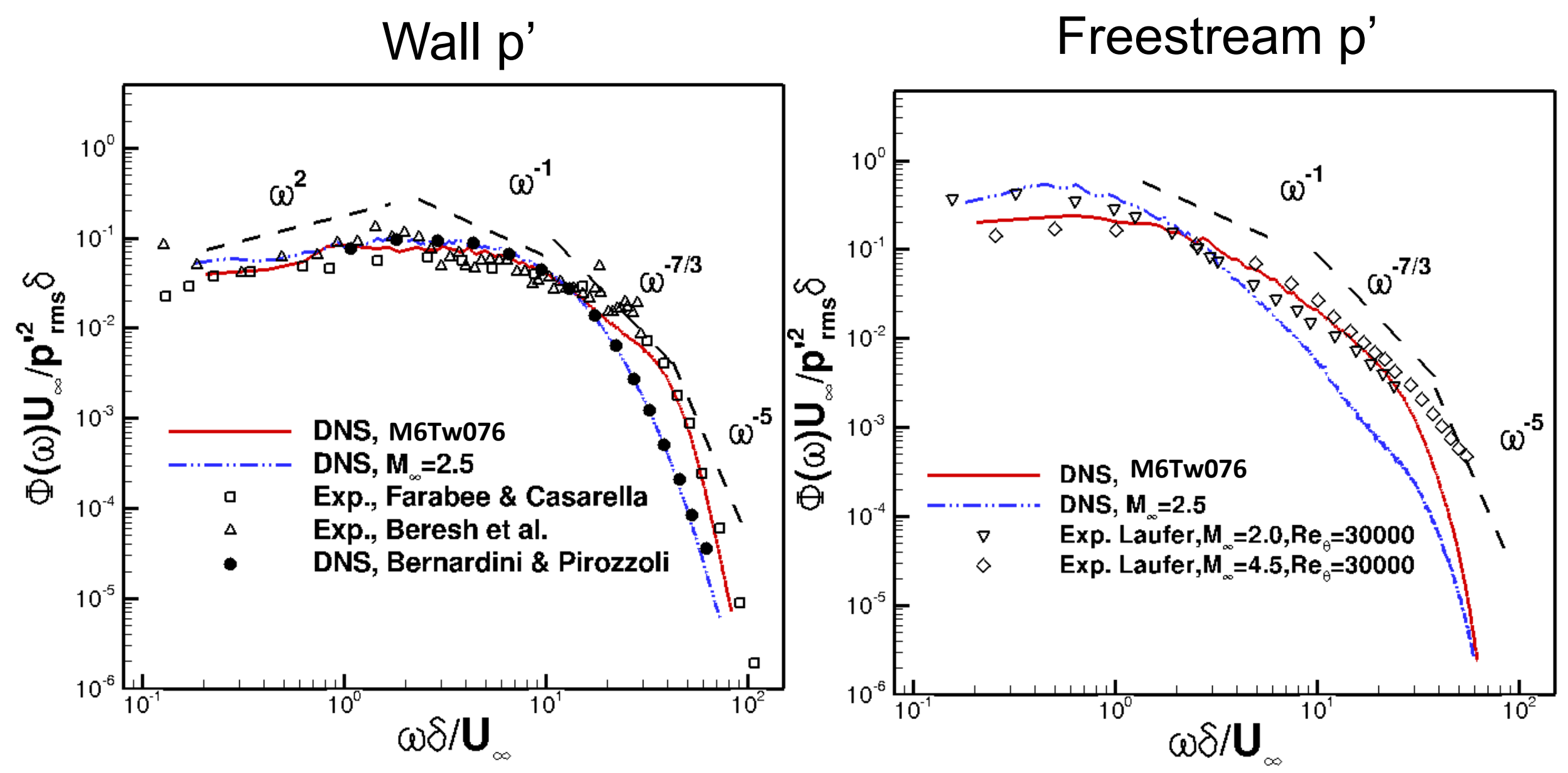


\section{DNS Setup Case M14Tw18}

$M_{\infty}=14, R_{\theta} \approx 13152$,

$\operatorname{Re}_{\mathrm{T}} \approx 633, \mathrm{~T}_{\mathrm{W}} / \mathrm{T}_{\mathrm{r}} \approx 0.18$

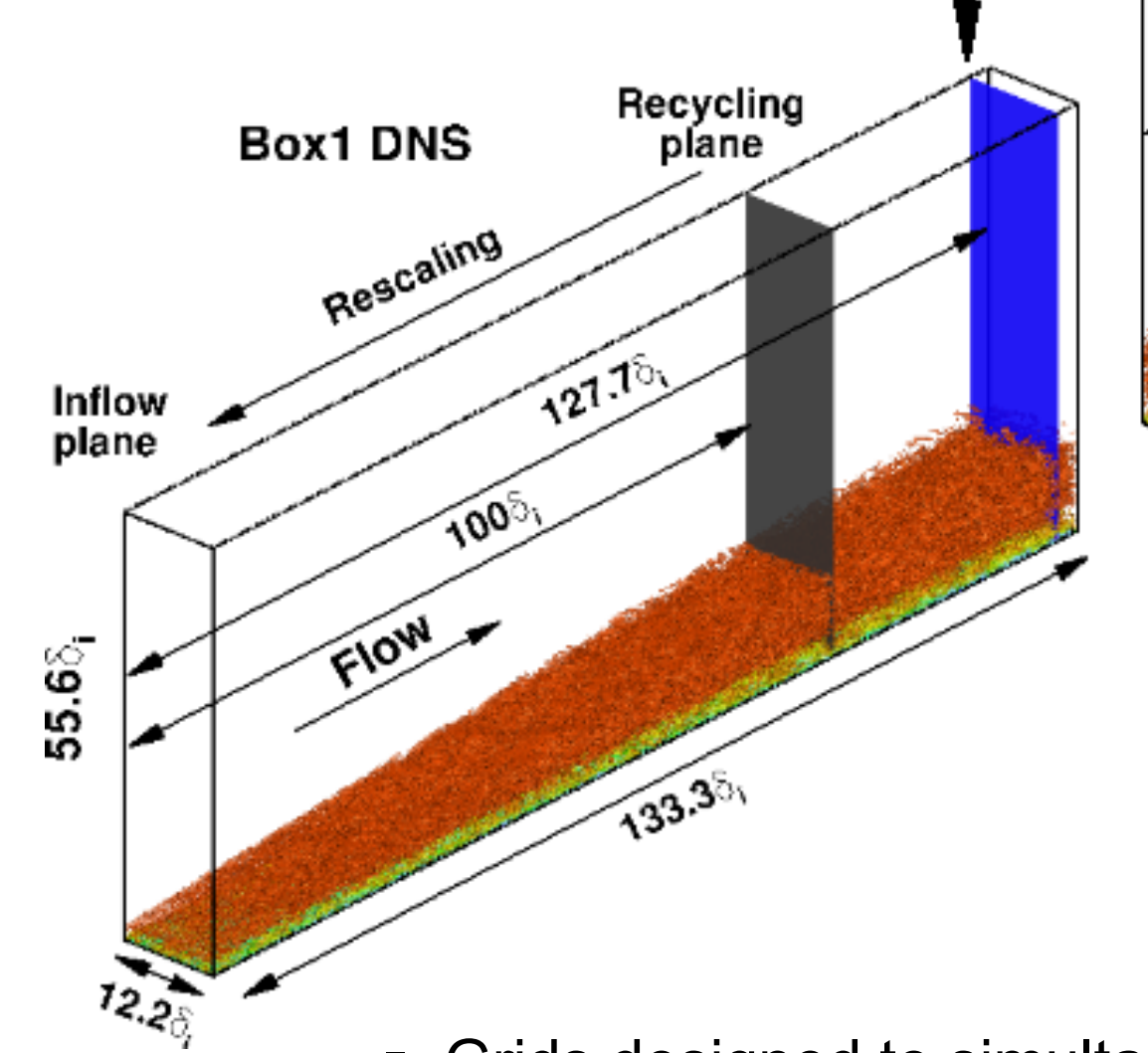

- Grids designed to simultaneously resolve both the hydrodynamic disturbances and near-acoustic field

- WENO (Jiang \& Shu 1996, Martin et al. 2007)

- Úniform grid in streamwise-spanwise direction:

- $\Delta \mathrm{x}^{+} \approx 9.4, \Delta \mathrm{y}^{+} \approx 4.7$

- $\Delta \mathrm{z}_{\mathrm{w}}{ }^{+} \approx 0.47, \mathrm{~N}_{\mathrm{z}}=19$ for $\mathrm{z}^{+}<10$,

- $\Delta z_{\delta}^{+} \approx 5.7, N_{z}=186$ for $z<\delta$

- $\mathrm{N}_{\mathrm{x}} \times \mathrm{N}_{\mathrm{y}} \times \mathrm{N}_{\mathrm{z}}=2500 \times 460 \times 540$ (Box 1 DNS)

- $N_{x} \times N_{y} \times N_{z}=1500 \times 460 \times 786$ (Box 2 DNS) 


\section{Numerical Schlieren Visualization M14Tw18}
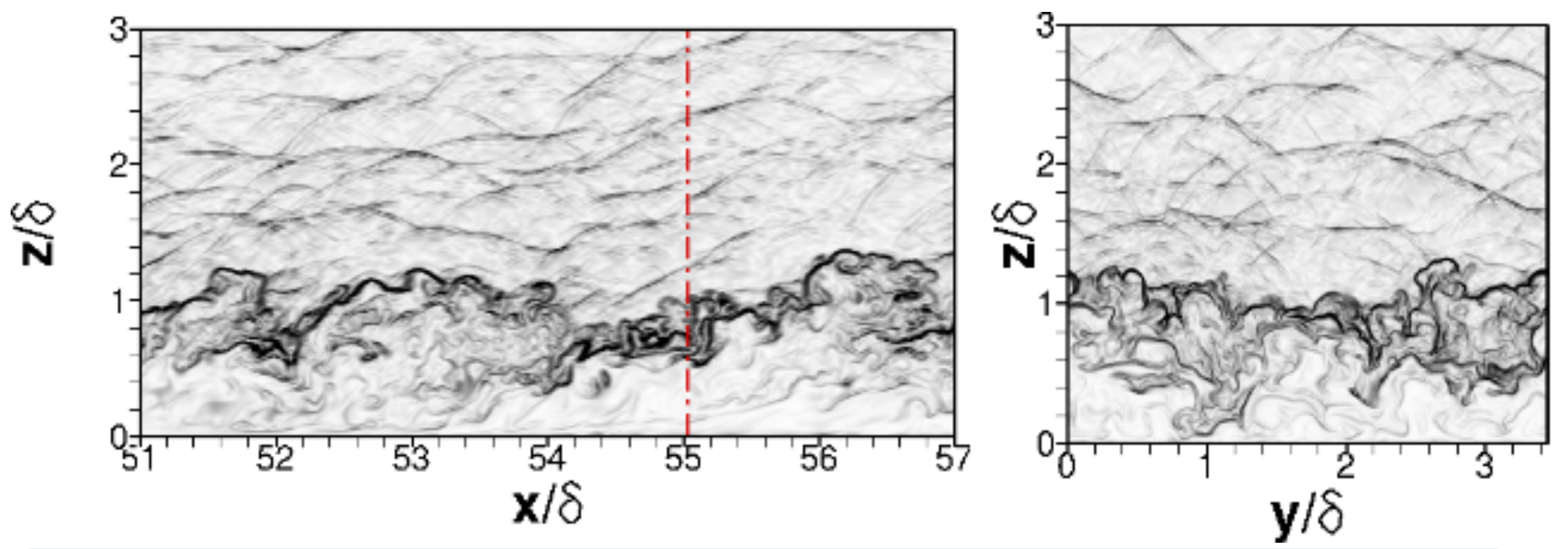

- Large scale motions cause incursions of the freestream irrotational flow into the boundary layer

- Distributed regions of strong density gradient can be seen within the boundary layer

- Existence of 'shocklets'??? 


\section{van Driest Transformed Mean Velocity Profile}

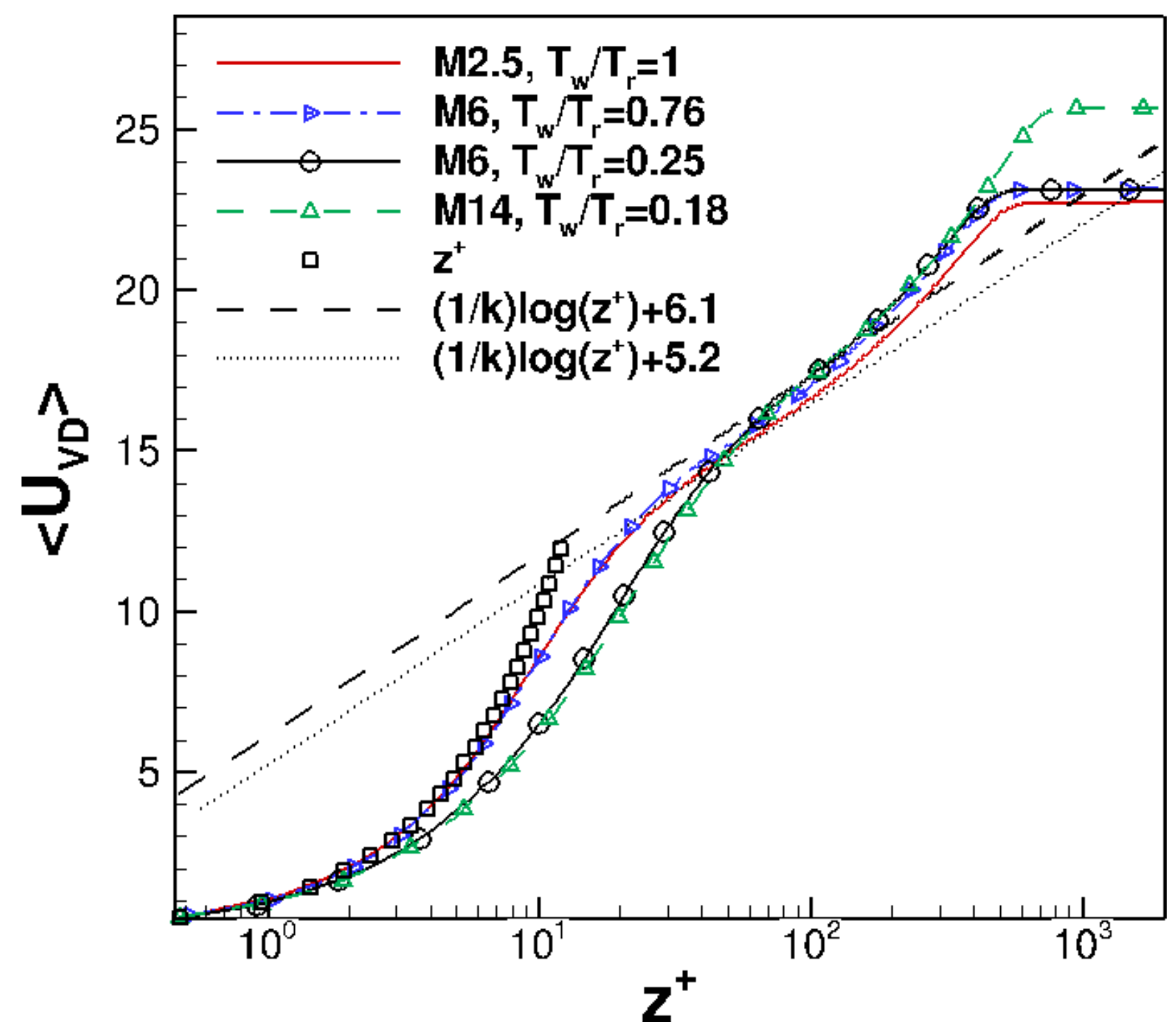




\section{Turbulence Intensities}
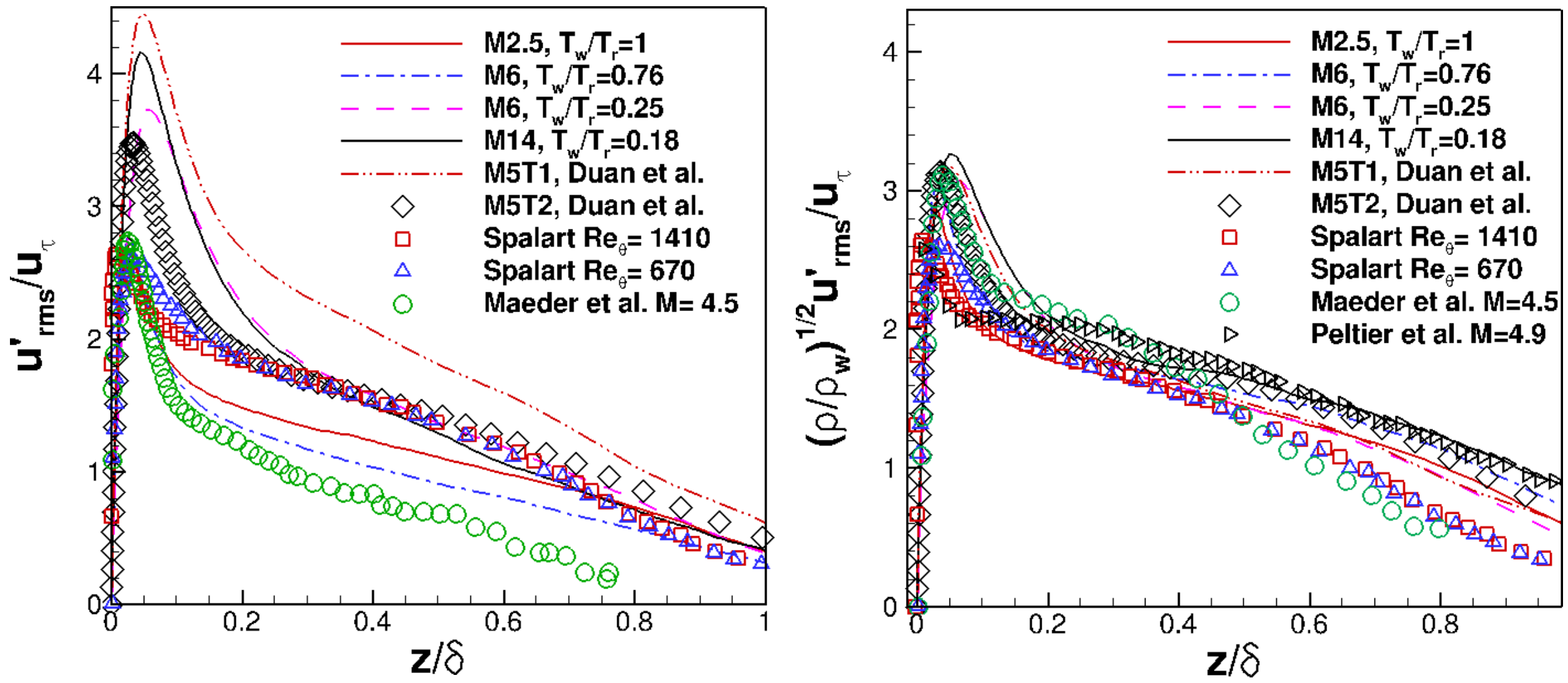

Significantly improved collapse of data is achieved by Morkovin's scaling 


\section{Turbulent Mach Number and Fluctuating Mach Number}
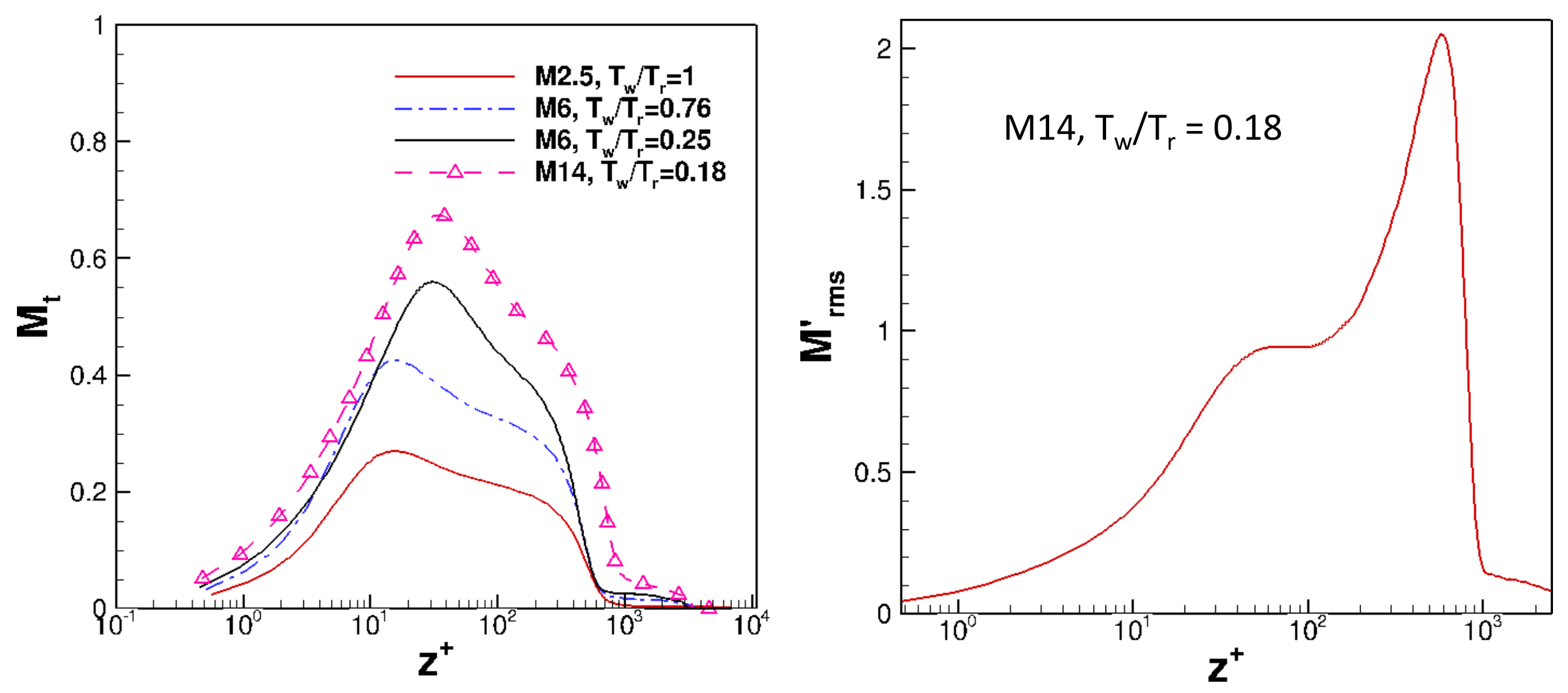


\section{Pressure Fluctuation Intensity}
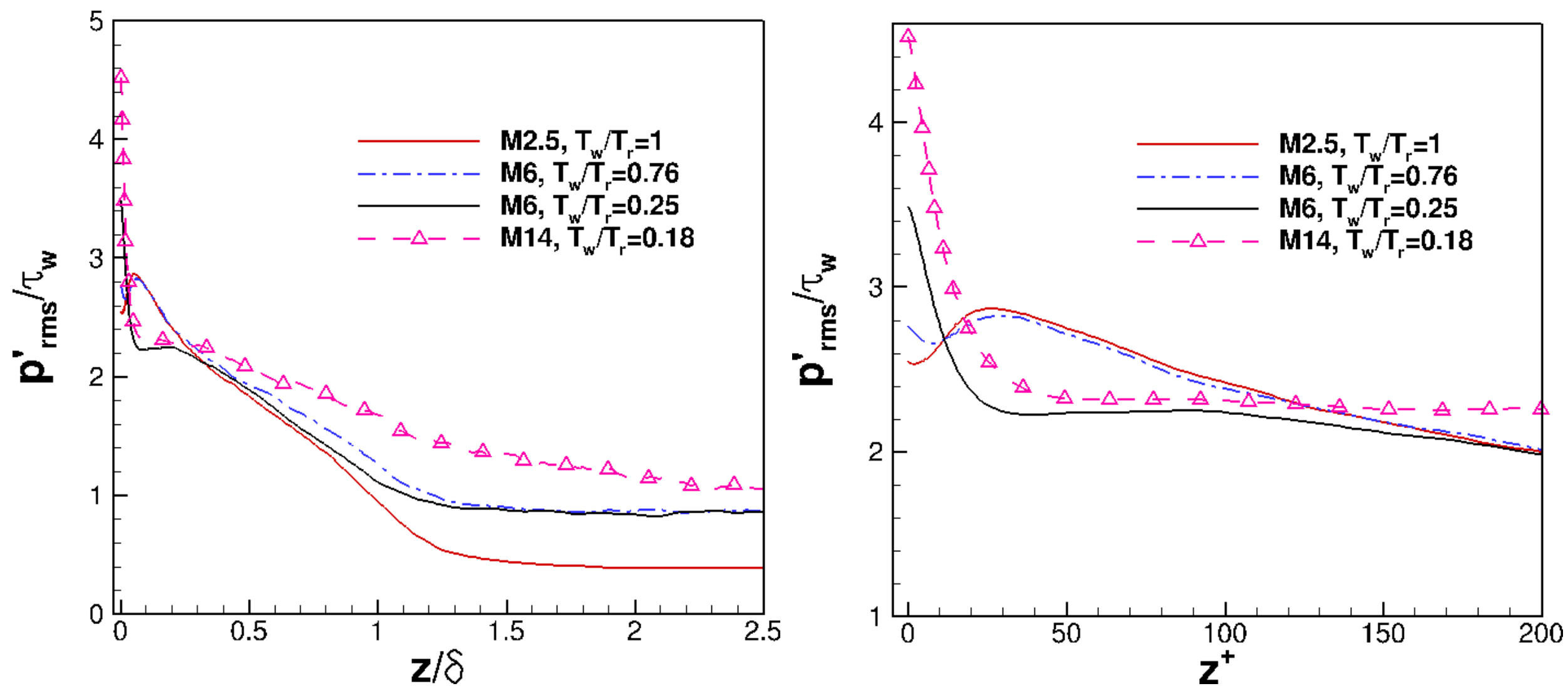

$\mathrm{p}^{\prime}{ }_{\mathrm{rms}} / \tau_{\mathrm{w}}$ near the wall shows a strong wall-temperature dependence

$\mathrm{p}^{\prime}{ }_{\mathrm{rms}} / \tau_{\mathrm{w}}$ in the free stream increases with Mach number and is insensitive to wall temperature 


\section{Pre-multiplied p' Frequency Spectra}

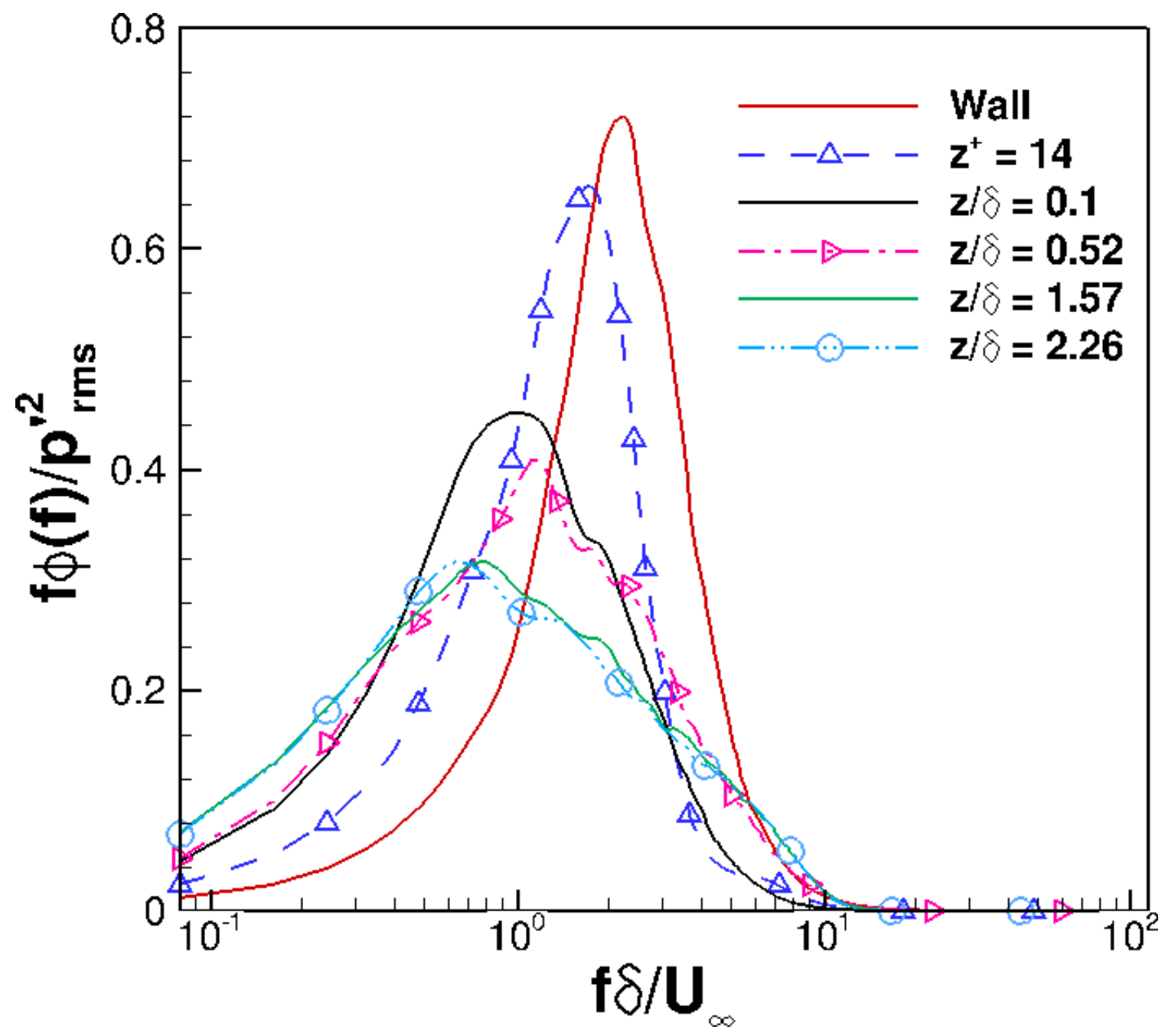

$p^{\prime}$ spectrum peak shifts to lower frequencies as the location of interest moves away from the wall 


\section{Pre-multiplied p' Frequency Spectra}

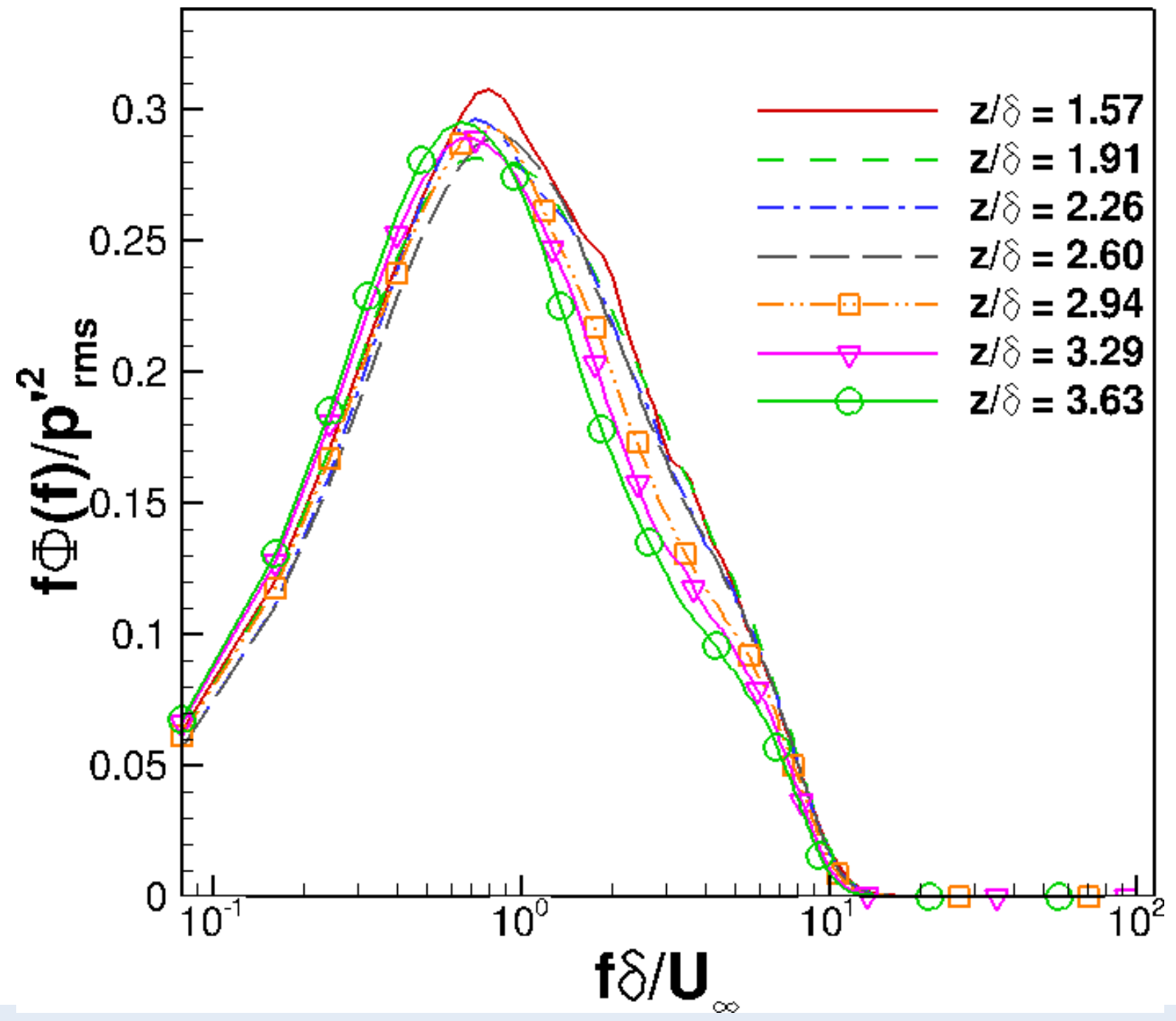

Good convergence of $p^{\prime}$ spectra in the free stream

Freestream $p^{\prime}$ spectrum centered at $f \delta / U_{\infty} \approx 0.7$ 


\section{Fluctuating Wall Quantities}

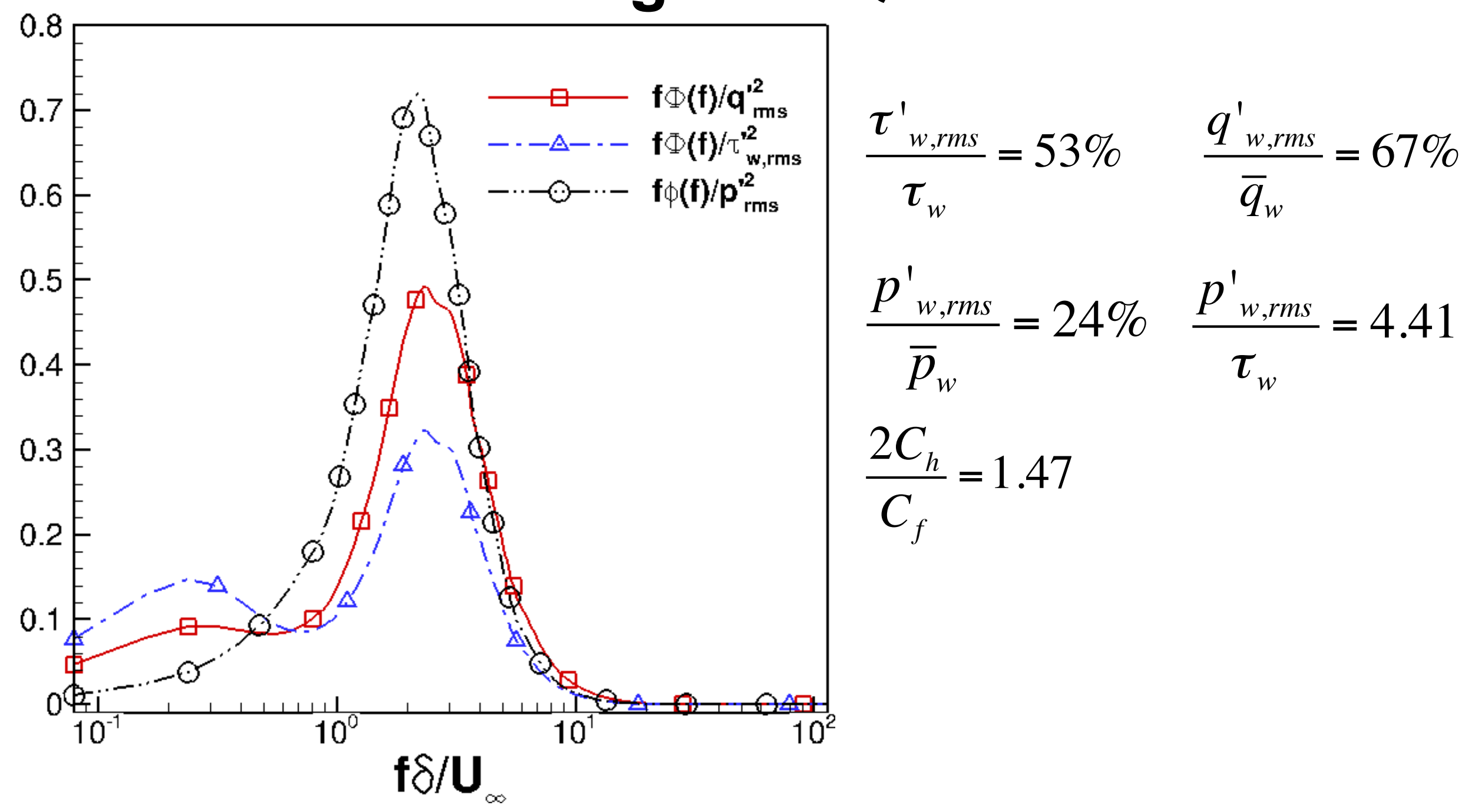

$p_{w}, \tau_{w}$, and $q_{w}$ show large fluctuations relative to the mean value $p^{\prime}{ }_{w} \tau^{\prime}{ }_{w}$ and $q^{\prime}{ }_{w}$ spectra peak at the same frequency of $\mathrm{f} \delta / \mathrm{U}_{\infty} \approx 2$ 


\section{Propagation Speed of Acoustic Disturbance}

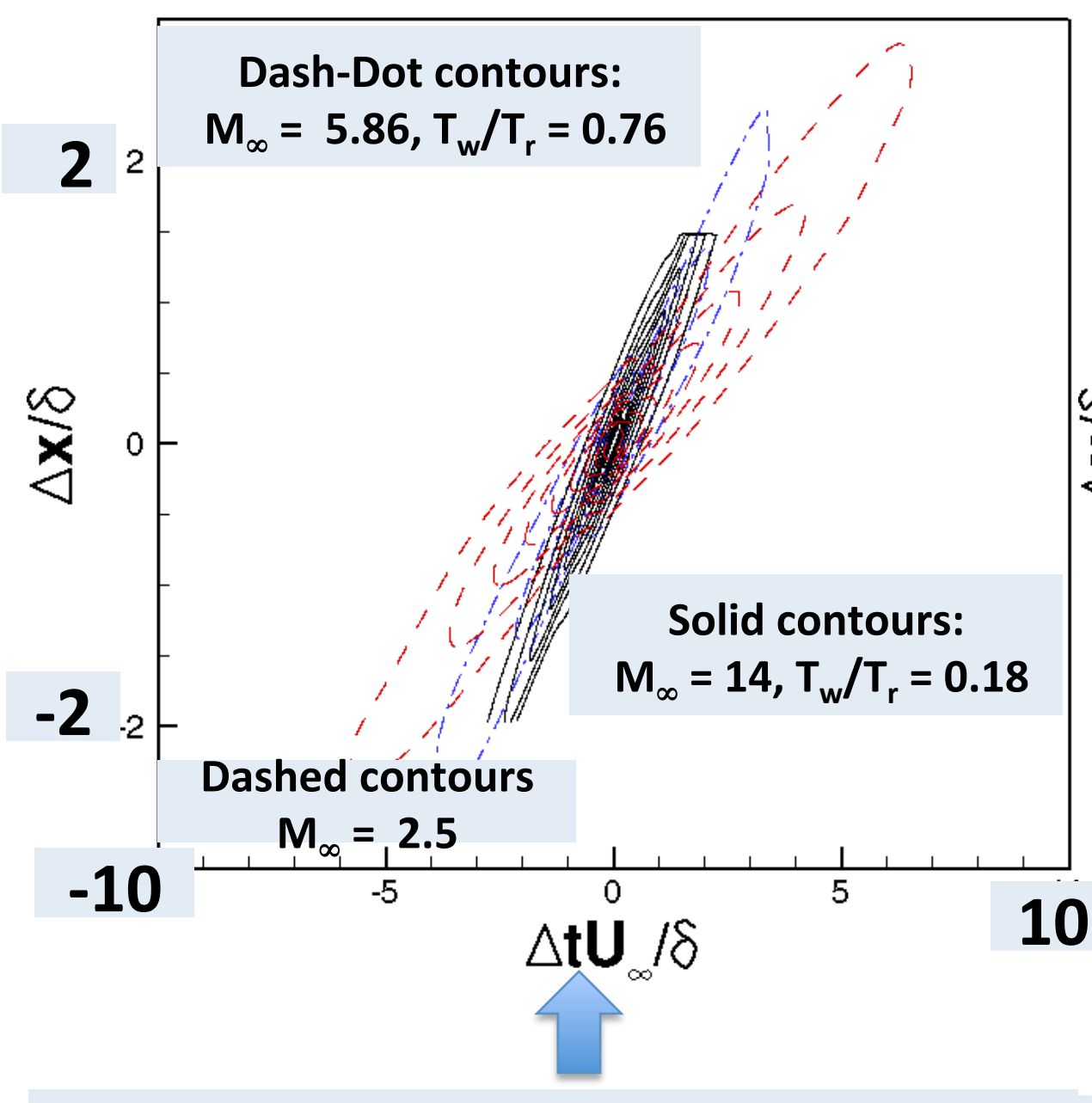

Faster propagation speed of freestream fluctuations as Mach number increases

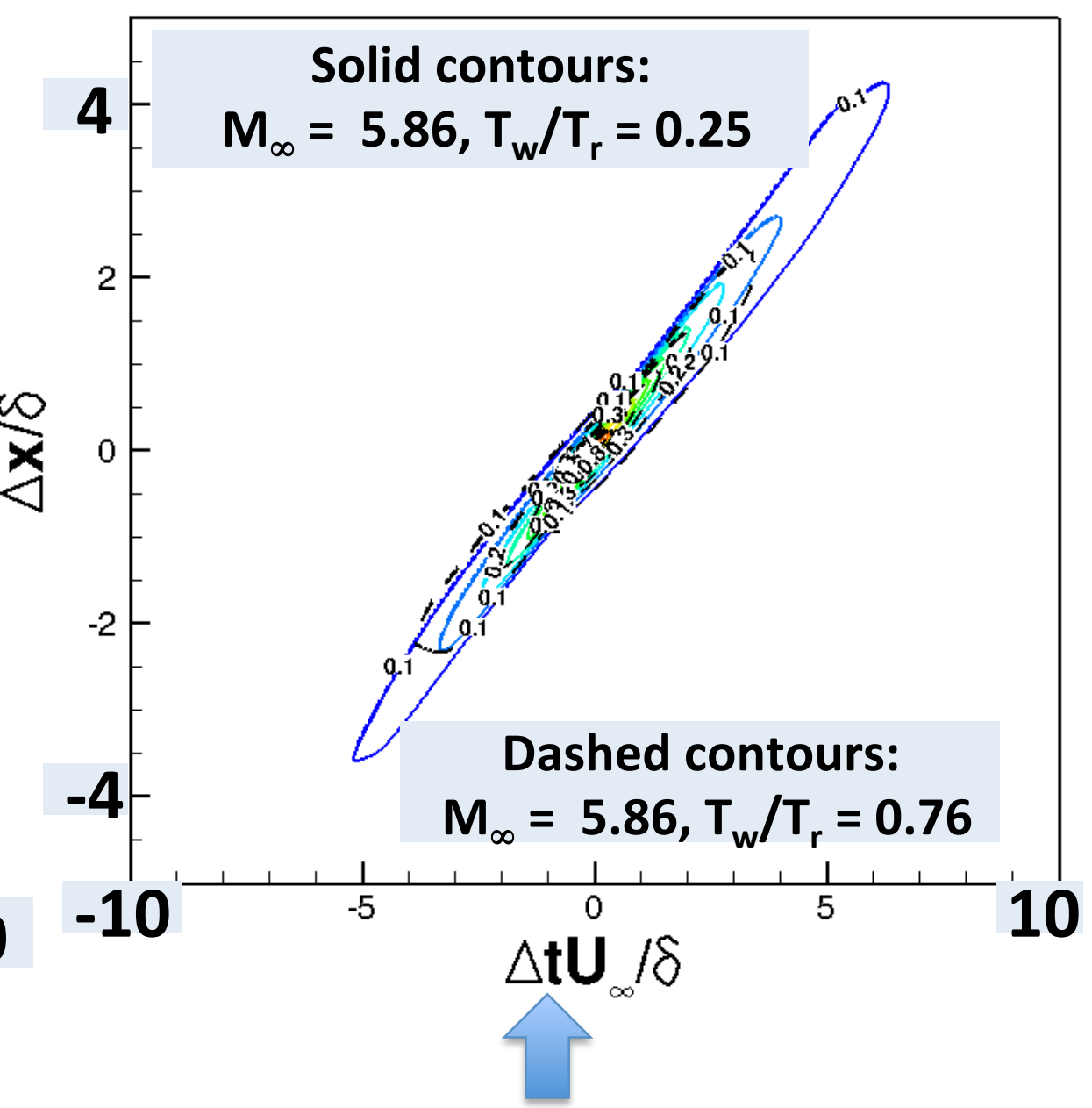

Wall Temperature has subtle influence on the propagation speed of freestream fluctuations 


\section{Numerical Schlieren Visualization}

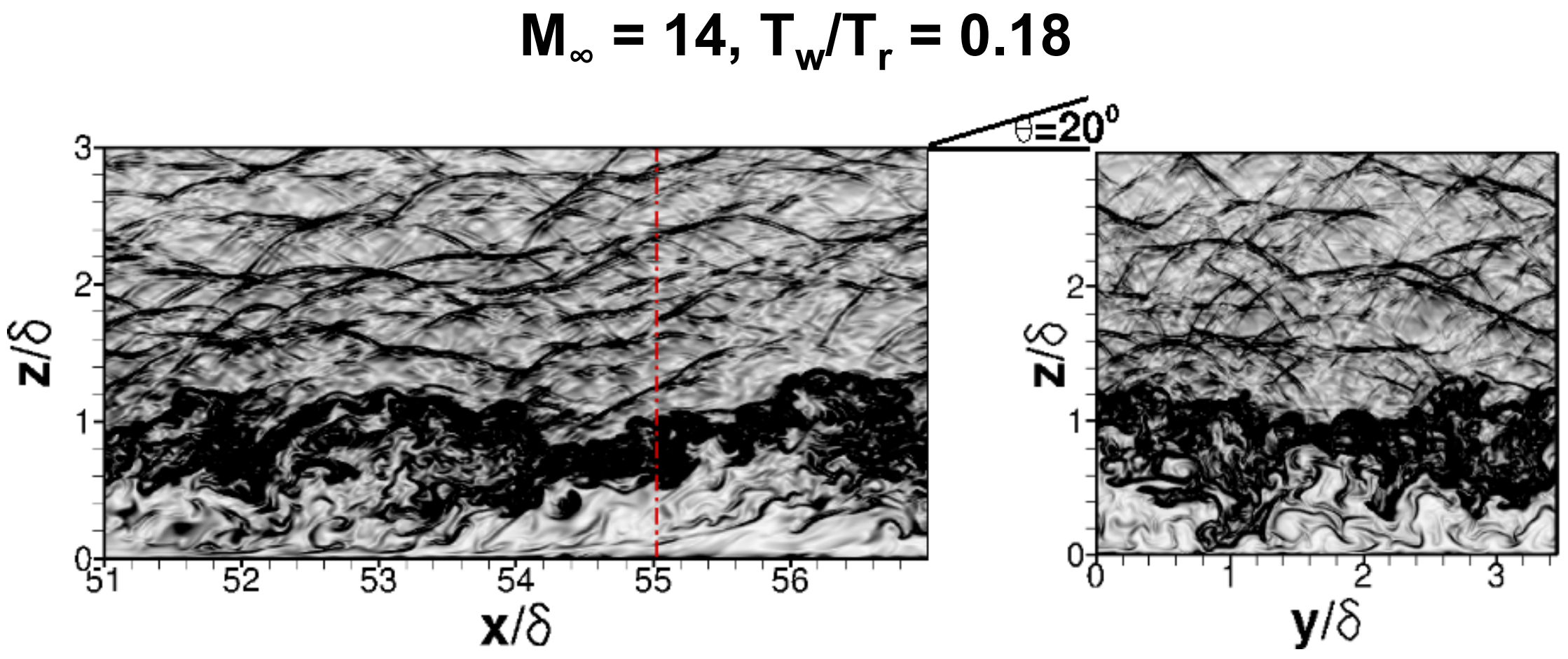

- Random

- Finite spatial coherence

- Preferred range of orientation for eddy Mach waves $>$ Higher inclination than Mach wave direction 


\section{Summary and Conclusion}

- Turbulence statistics and pressure fluctuations induced by a Mach 14 turbulent boundary layer were investigated

- $M_{\infty}=14, \operatorname{Re}_{\tau} \approx 633, T_{w} / T_{r}=0.18$ (a condition of AEDC Tunnel 9)

- Velocity fluctuations scales according to the Morkovin's scaling

- Property of pressure fluctuations varies dramatically as a function of wall-normal distance within the inner layer $\left(\mathrm{z} / \delta<0.08\right.$ or $\left.\mathrm{z}^{+}<50\right)$

- fluctuation magnitude $\mathrm{p}_{\mathrm{rms}} / \tau_{\mathrm{w}}$

- dominant frequency $f_{p k}$ associate with pressure spectrum

- Fluctuating wall quantities ( $\left.\mathrm{p}_{\mathrm{w}}, \tau^{\prime}{ }_{\mathrm{w}}, \mathrm{q}^{\prime}{ }_{\mathrm{w}}\right)$

- Large fluctuation amplitude relative to the mean values $\left(\mathrm{p}_{\mathrm{rm}}{ }_{\mathrm{rm}} / \mathrm{p}_{\mathrm{w}}=24 \%, \tau_{\mathrm{w}, \mathrm{rms}}{ }^{\prime}\right.$ $\tau_{\mathrm{w}}=53 \%, \mathrm{q}_{\mathrm{w}, \mathrm{rms}}^{\prime} / \mathrm{q}_{\mathrm{w}}=67 \%$ )

- A match in dominant frequency among $\mathrm{p}^{\prime}{ }_{\mathrm{w}}, \tau{ }_{\mathrm{w}}, \mathrm{q}^{\prime}{ }_{\mathrm{w}}$ with $\mathrm{f} \delta / \mathrm{U}_{\infty} \approx 2$

- Freestream pressure fluctuations involves a broadband peak centered at $f \delta / \mathrm{U}_{\infty} \approx$ 07 


\section{Outlook}

\section{Facility Disturbance + Receptivity}
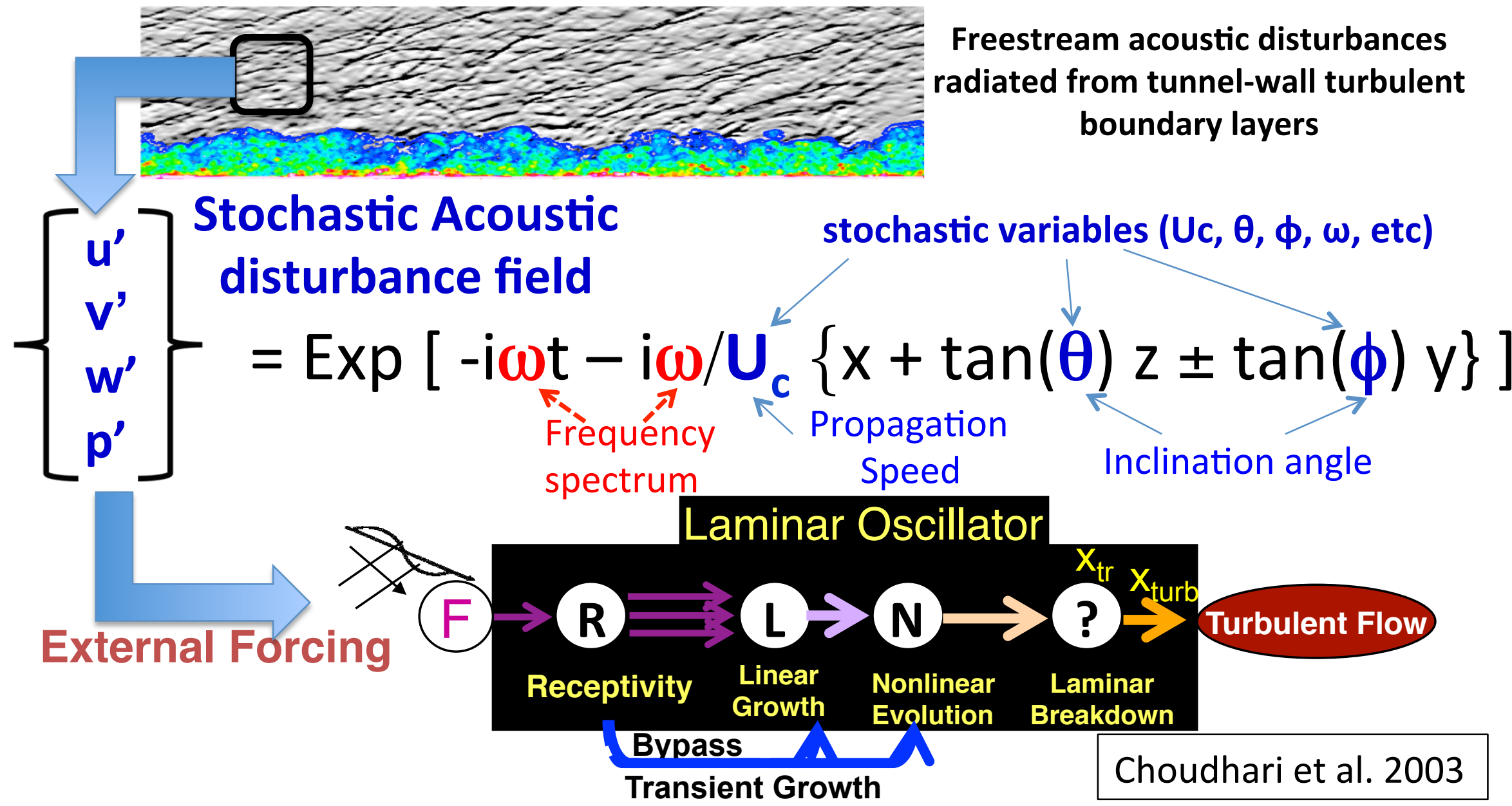

Provide "practical" input data regarding disturbance environment for conducting stability analysis in the context of actual wind-tunnel experiments Enable holistic prediction of transition in High-Speed Boundary Layers 


\section{Acknowledgment}

- Prof. Steve Schneider and Dr. Katya Casper

- for providing wind-tunnel measurements for comparison with DNS

- Financial Support from AFOSR (Award No.

FA9550-14-1-0170)

- Support from NASA Langley (Cooperative Agreement No. NNL09AA00A) and DARPA to initiate the project

- Computational resources provided by the DoD High Performance Computing Modernization Program and the NASA Advanced Supercomputing Division 